\title{
Acute transverse myelitis attributed to SARS-CoV-2 infection presenting as impaired mobility: a case report
}

\author{
Sarah Kilbertus ${ }^{1}$ (D)
}

Received: 6 December 2020 / Accepted: 9 February 2021 / Published online: 1 March 2021

(C) The Author(s), under exclusive licence to Canadian Association of Emergency Physicians (CAEP)/ Association Canadienne de Médecine d'Urgence (ACMU) 2021

Keywords Transverse myelitis · COVID-19 · SARS-CoV-2 $\cdot$ Neurologic dysfunction

\section{Introduction}

Transverse myelitis (TM) is a rare inflammatory condition of the spinal cord with an estimated incidence of 1-8 cases per million per year. It can present with rapid onset weakness, sensory alterations and/or bladder or bowel dysfunction, and is often attributed to an infectious etiology, or secondary to an autoimmune disorder such as multiple sclerosis [1]. As the COVID-19 pandemic has progressed, we have learned that the SARS-CoV-2 virus can cause a variety of neurological manifestations including anosmia, dysgeusia, Guillain-Barre syndrome and stroke [1]. Here we present a case of acute TM attributed to infection by the SARS-CoV-2 virus.

\section{Case presentation}

A 46-year-old previously healthy male presented to the emergency department (ED) with concerns of generalized body stiffness which began $24 \mathrm{~h}$ prior. His symptoms had progressed to the point that they impaired his ability to mobilize and transfer independently and he felt his gait was abnormal. Prior to the onset of these symptoms he had felt well, apart from a low-grade fever of $37.5^{\circ} \mathrm{C}$ he incidentally measured at home the day before. He denied any headache, neck pain, back pain, vision changes, confusion, chest pain, cough or shortness of breath. There was no history of trauma, recent illness or sick contacts. He described

Sarah Kilbertus

sarah.kilbertus@mail.utoronto.ca

1 Department of Emergency Services, Sunnybrook Health

Sciences Centre, 2075 Bayview Avenue, suite C753,

Toronto, ON M4N 3M5, Canada his symptoms to be more of a feeling of stiffness and hyperesthesia in his lower legs, rather than true weakness or paresthesias.

His vital signs in the ED included a temperature of $35.9^{\circ} \mathrm{C}$, a heart rate of 97 beats per minute, a respiratory rate of 18 breaths per minute, a blood pressure of $150 / 95 \mathrm{mmHg}$ and an oxygen saturation of $97 \%$ on room air. Physical exam demonstrated normal cranial nerves, $5 / 5$ power to all limbs except for bilateral knee extension at $4 / 5$, and normal sensation. He was hyperreflexic in the upper and lower extremities with sustained clonus in his ankles. He required assistance in mobilizing from the stretcher due to truncal weakness, and he was ataxic with a wide-based gait on ambulation testing. Although his rectal tone was normal, he had urinary retention with a post void residual of $337 \mathrm{~mL}$. Laboratory results including a CBC, electrolytes, renal and liver function, CK and TSH were unremarkable. Inflammatory markers were mildly elevated with a CRP of $6 \mathrm{mg} / \mathrm{L}$ and ESR of $28 \mathrm{~mm} / \mathrm{h}$.

An unenhanced CT head was performed which did not demonstrate any acute intracranial abnormalities. An expedited MRI of the brain and spine demonstrated an abnormal T2 high signal within the cervical cord extending from $\mathrm{C} 3$ to $\mathrm{T} 1$ in keeping with cord edema. There was also a C4-5 central disc protrusion causing mild left-sided compression of the cervical cord and a C5-6 disc protrusion with significant cervical cord compression. Neurosurgery was consulted for an opinion regarding the need for spinal decompression; however, they did not feel the patient's symptoms were in keeping with a diagnosis of cervical stenosis. Neurology and internal medicine were consulted to consider an alternate diagnosis and the patient was admitted to hospital.

A nasopharyngeal swab was sent for detection of SARS$\mathrm{CoV}-2$ as a routine measure for all patients admitted to hospital. The swab came back positive 3 days after the patient was originally assessed in the ED. A lumbar puncture was 


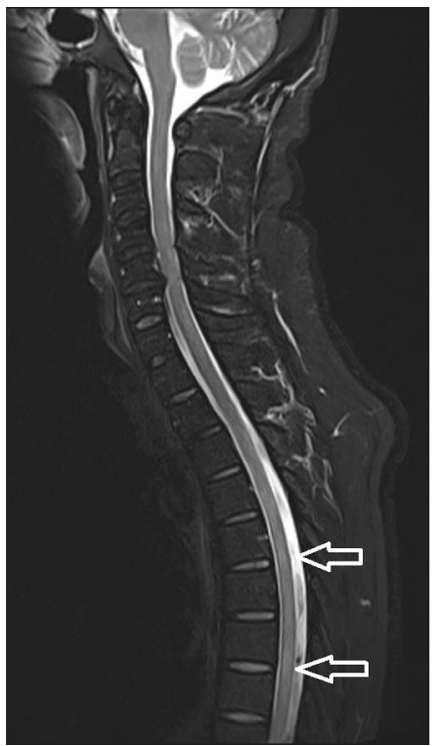

Fig. 1 MRI (T2WI) sagittal spine showing hyperintensities (arrows) in the spinal cord corresponding to T5 and T7 vertebra suggestive of acute transverse myelitis

performed by neurology which demonstrated an elevated CSF WBC count of $50 \times 10^{6} / \mathrm{L}$, a lymphocyte count of $41 \times 10^{6} / \mathrm{L}$, a neutrophil count of $3 \times 10^{6} / \mathrm{L}$, an elevated protein of $502 \mathrm{mg} / \mathrm{L}$ and a normal glucose. The culture did not demonstrate any growth and viral PCR for HSV and VZV was negative. A thorough infectious disease and autoimmune work up was also completed to rule out other etiologies and was non-contributory. Due to progression of symptoms, a repeat MRI was done on day 3 of admission, which demonstrated extension in the degree of T2/FLAIR signal change within the cord which now extended from the upper cervical spine to the conus medullaris (see Fig. 1). A presumptive diagnosis of TM due to SARS-CoV-2 was made and the patient was started on Methylprednisolone $1 \mathrm{~g} \mathrm{IV}$ for 5 days.

The patient was eventually discharged to a rehab facility after showing improvements in his weakness after a 15 day stay in hospital. At the time of discharge, he was able to ambulate with a 4-wheel walker and sit unsupported while still having difficulty with urination. He never developed any additional SARS CoV-2 symptoms.

\section{Discussion}

To our knowledge, this is the first reported case of TM as part of the COVID-19 illness in Canada. Other cases have been reported around the world including in China, the
United States, Australia, India, Europe and the Middle East [1-5]. Bladder dysfunction presenting as retention or incontinence was a universal finding among all case reports and is congruent with what our patient experienced. Common CSF findings in patients who received a lumbar puncture typically demonstrated elevated lymphocytes and protein, and a normal glucose. Interestingly, however, symptoms and physical exam findings were markedly different across case reports. Patients could present with sensory disturbances, motor impairment, or both; reflexes could be hypoactive or hyperactive; weakness ranged from very mild to total flaccid paralysis; some had fever and symptoms of an upper respiratory tract infection (URTI) prior to the onset of their neurological symptoms, while others did not; and in those who's illness began with URTI symptoms, it took anywhere from 2 to 18 days for neurological signs to manifest [1-5]. These differences in presentation of COVID-19 TM serve to highlight the complex nature of COVID-19 as a disease entity.

Emergency clinicians should consider COVID-19 TM in any patient presenting with neurological complaints such as limb weakness and/or paresthesias and new changes to bowel or bladder function, to expediate appropriate referral and treatment. Preliminary evidence suggests that treatment with intravenous steroids and plasma exchange can improve symptoms if started early [1-5], making it of paramount importance to obtain imaging and neurological consultation without delay. As physicians on the front lines, we should also consider earlier isolation and testing for SARS CoV-2 in patients presenting with neurological symptoms, even in the absence of the more typical URTI symptoms [5].

\section{Compliance with ethical standards}

Conflict of interest The author declares that they have no conflict of interest.

\section{References}

1. Valiuddin H, Skwirsk B, Paz-Arabo P. Acute transverse myelitis associated with SARS-CoV-2: a case-report. Brain Behav Immun Heal. 2020;5:100091.

2. Zhao K, Huang J, Dai D, Feng Y, Liu L, Nie S. Acute myelitis after SARS-CoV-2 infection: a case report. MedRxiv. 2020;3:16.

3. Sarma D, Bilello L. A case report of acute transverse myelitis following novel coronavirus infection. Clin Pract Cases Emerg Med. 2020;4(3):321-3.

4. Chow CCN, Magnussen J, Ip J, Su Y. Acute transverse myelitis in COVID-19 infection. BMJ Case Rep. 2020;13(8):1-4.

5. Chakraborty U, Chandra A, Ray AK, Biswas P. COVID-19-associated acute transverse myelitis: a rare entity. BMJ Case Rep. 2020;13:8 